High Energy Phenomena in Relativistic Outflows III (HEPRO III)

International Journal of Modern Physics: Conference Series

Vol. 8 (2012) 327-330

(C) World Scientific Publishing Company

DOI: $10.1142 / \mathrm{S} 2010194512004813$

\title{
BL LAC POPULATION STUDY: EARLY RESULTS USING THE Fermi 11-MONTH CATALOG
}

\author{
LUCIE GÉRARD, SANTIAGO PITA and MICHAEL PUNCH \\ Université Denis Diderot, Laboratoire APC, 10 rue Alice Domon et Léonie Duquet \\ 75205 Paris Cedex 13, France \\ lucie.gerard@apc.univ-paris7.fr, santiago.pita@apc.univ-paris7.fr,punch@in2p3.fr
}

\section{GILLES HENRI}

Institut de Planétologie et d'Astrophysique de Grenoble

BP 5338041 Grenoble Cedex 9, France

Gilles.Henri@obs.ujf-grenoble.fr

\begin{abstract}
In the framework of AGN unification, BL Lacs and their parent population would share the same intrinsic characteristics, the observational differences being due to the orientation of the jet compared to our line of sight. BL Lacs would be those objects whose jet is oriented towards us, Doppler boosting the emission. The growing number of BL Lacs detected at $\mathrm{HE}(>100 \mathrm{MeV})$ and $\mathrm{VHE}(>100 \mathrm{GeV})$ is a challenge for this scheme, since the high values of Doppler factors needed to explain the emission of these sources imply a large density for the parent population.

We studied the BL Lac source sample detected by Fermi after 11 months of observation. Using the data presented in Fermi's first AGN catalog, we put constraints on the intrinsic characteristics of this BL Lac population, such as the intrinsic luminosity and Lorentz factor distributions. Based on these results, we used Monte Carlo simulations to constrain the space density of the parent population and the jet opening angle.
\end{abstract}

Keywords: AGN; BL Lac; Fermi; ACT

\section{Introduction}

One of the key issues of the study of AGNs is to explore the possibility to organize the different classes of objects within a unified vision. In this unification scheme, AGNs would share the same intrinsic characteristics and the observed differences would be due to the orientation of those highly anisotropically emitting objects compared to our line of sight. In the particular case of blazars, the relativistic jet would be directed towards us, Doppler boosting the emission and the parent population would be composed of the objects whose jet is directed away from us.

In the simplest emission models which generally reproduce fairly well the observed average emission of most AGNs, the opening angle of the jet depends only on the relativistic beaming - this angle decreasing as the Lorentz factor increases. In this context, the growing number of blazars detected at $\mathrm{HE}(>100 \mathrm{MeV})$ and VHE $(>100 \mathrm{GeV})$ - mainly BL Lac at VHE energies - implies a large density for 
the parent population. Indeed, the high values of Doppler factors needed to explain the emission of these sources at such energies require large Lorentz factors and a jet directed close to our line of sight. The probability to see a blazar is then very much reduced, leading to the so-called "Doppler factor crisis" ${ }^{1}$ which denotes the contradiction between the high values of Doppler factor needed in the simple one zone emission model and those, much smaller, inferred from the unification between blazars and radio-galaxies. The BL Lacs detected at HE and VHE are used to test the unification model. The large population detected by Fermi is mainly used to constrain some of the intrinsic characteristics of these objects before using Monte Carlo simulations to investigate the density of the parent population and the jets of these objects whose geometry is still poorly known.

\section{Presentation of the study and preliminary results}

The spectral energy distributions (SEDs) of blazar-type AGNs are characterized by a two-bump structure. This study focuses on the average emission of the peak located at the highest energies, partially covered with Fermi and the Atmospheric Cherenkov Telescopes (ACT). For BL Lacs, the observed emission is Doppler boosted; it is also completely characterized by the shape of the SED and, by the energy position $\left(E_{p}\right)$ and the luminosity $\left(L_{p}\right)$ of the peak. The boost doesn't affect the shape of the SED but simply shifts it in energy and luminosity and so it is possible to access intrinsic characteristics of the population by working in the $E_{p}-L_{p}$ plane.

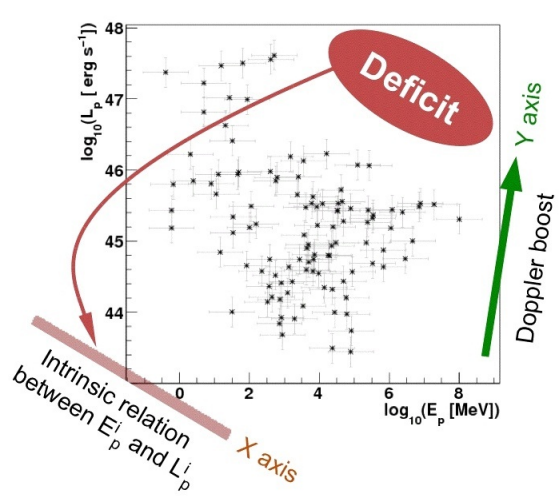

Fig. 1. Peak luminosity and energy position of the BL Lacs detected by Fermi, with known redshift. The errors represent the uncertainties in the determination of $L_{p}$ and $E_{p}$.

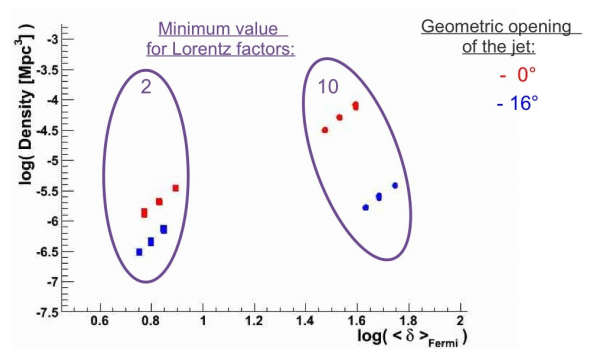

Fig. 2. Parent population density vs. the Doppler factor mean value of the AGN simulated as detectable by Fermi. 


\subsection{Intrinsic characteristics of Fermi's BL Lac population}

The study is limited to the BL Lac population detected by Fermi whose redshift is known and below 0.5. We use the fluxes and the spectral indexes presented in the Fermi 11-month catalog ${ }^{2}$. To estimate $E_{p}$ and $L_{p}$ for each BL Lac, we assume a relation between $E_{p}$ and the spectral index measured by Fermi ${ }^{3}$ and a unique empirical spectral shape - a broken power law with curved transition - whose only ambition is to account for the fluxes measured at HE and VHE.

Figure 1 features the $E_{p}$ and $L_{p}$ of the BL Lacs detected by Fermi used in this study. A deficit at high $E_{p}$ and high $L_{p}$ is observed. Neither the Doppler boost translating the objects along the green axis represented in figure 1, named " $Y$ axis" - nor Fermi's sensitivity can explain this effect. On the other hand, such an effect would be expected in the case of an intrinsic relation between the values $E_{p}$ and $L_{p}$, namely $E_{p_{i}}$ and $L_{p_{i}}$ where the $i$ stands for "intrinsic". This relation is symbolized by the direction of the red axis in figure 1 , named " $X$ axis".

We assume this intrinsic relation - expected in case of a the blazar sequence ${ }^{4}$ - and, from the distribution of BL Lacs in the $E_{p}-L_{p}$ plan represented in figure 1, estimate the link between $E_{p_{i}}$ and $L_{p_{i}}$ to be such as $\ln \left(E_{p_{i}}\right)=-0.45 \ln \left(L_{p_{i}}\right)+$ $C$, where $C$ is an unknown constant. The distribution of intrinsic luminosities is obtained by canceling the boost effect: projecting the objects along the Doppler boost axis onto the $\mathrm{X}$ axis and taking Fermi's horizon into account. The objects distributed along the direction of the assumed intrinsic relation are supposed to have the same Doppler factor value. Considering this effect, the Doppler factor distribution is constructed, using the same method: now projecting the objects along the $\mathrm{X}$ axis onto $\mathrm{Y}$ axis. Both the intrinsic luminosity and Doppler factor distributions are compatible with power laws: $\mathrm{d} N / \mathrm{d} L_{p_{i}} \propto L_{p_{i}}^{-1.75 \pm 0.4}$ and $\mathrm{d} N / \mathrm{d} \delta \propto$ $\delta^{-2.8 \pm 0.4}$ respectively. The slope of the distribution of Doppler factors excludes a unique Lorentz factor for the entire population and is used to constrain their distribution. This results were obtained considering only the BL Lacs with $z^{6} 0.5$ in order to guard against bias due to a possible under representation of the objects with $z>0.5$ in Fermi's sample ${ }^{2}$.

\subsection{Monte Carlo simulation of parent populations}

In this section, we test the unification scheme with Monte Carlo simulations using the previous working hypothesis and constraints. The parent populations are simulated for different values for the geometric opening angles of the jet, minimal values of Lorentz factors and intrinsic positions of the objects in the $E_{p}-L_{p}$ plane - these are the free parameters of the simulations. Objects are simulated as being isotropically distributed up to a redshift of 0.5 , with two symmetrical jets randomly oriented compared to our line of sight. The effects of Doppler boost and red-shift of the SED are simulated using the following relations: $E_{p}=\frac{\delta}{1+z} E_{p_{i}} ; \quad L_{p}=\delta^{p} L_{p_{i}}$ where $\delta$ is the Doppler factor, $z$ is the redshift and $p$ is equal to 3 which correspond to a continuous jet flow. For each AGN the flux in the energy band covered by Fermi is 
calculated and compared to the TS $=25$ flux limit of the instrument ${ }^{2}$ in order to select the detectable objects.

To determine the valid sets of free parameters, we compare, the distributions of objects simulated as detectable by Fermi to the distribution of those experimentally detected by Fermi. To do this, the total number of objects simulated as detectable by Fermi is normalized to the experimental value. The $E_{p}-L_{p}$ plane is divided in bins, whose size depends on the uncertainties in the determination of the experimental values of $L_{p}$ and $E_{p}$. The likelihood between the simulated and experimental distributions of objects in the $E_{p}-L_{p}$ plane is calculated using the Poisonian probability law associated to the number of objects in each bin.

The BL Lac population detected by Fermi could be reproduced with a single parent population with jets randomly oriented compared to our line of sight. The density of the simulated parent population - normalized in order to account for the number of BL Lacs detected by Fermi - and the mean value of the Doppler factors corresponding to the population of AGNs simulated as detectable by Fermi are the results of the simulations. Figure 2 shows this results for those simulations with the best likelihood. Various sets of free parameters are acceptable and experimental constraints on the density of the parent population and the Doppler factors of the objects detected by Fermi are needed to remove the free parameters degeneracy. This density has to be considered as a very conservative lower limit, calculated above the intrinsic luminosity value below which no constraint is available because it would not be possible to detect AGN with Fermi's 11-month sensitivity.

\section{Conclusions}

Some intrinsic characteristics of the BL Lac population has been constrained using the Fermi 11-month catalog. As expected within the framework of AGN unification, Monte Carlo simulations have shown that it is possible to account for the BL Lacs detected by Fermi with a single parent population. To further constrain the jet geometry of these objects one needs to consider the experimental density of the parent population. Since only few non-blazars are detected at HE and VHE, this density has to be looked for at other wavelength where the emission is dominated by the isotropic component which will have to be taken into account in the simulations. This work is to be continued with Fermi's second AGN catalog.

\section{References}

1. G. Henri and L. Saugé, Astrophys. J. 640, 185 (2006).

2. A. A. Abdo, et al., Astrophys. J. 715, 429 (2010).

3. A. A. Abdo, et al., Astrophys. J. Suppl. 188, 405 (2010).

4. P. Padovani, Astrophys. Space Sci. 309, 63 (2007). 